\title{
Red light warning sign for clear-cell renal cell carcinoma-REDECT reveals imaging biomarker
}

$\mathrm{T}$ he ability to differentiate between indolent or benign renal cell tumours and the aggressive clearcell renal cell carcinoma (ccRCC) before surgical resection is important in clinical decision making. In terms of treatment options-and requirements-the type of tumour being treated is crucial. For example, for patients with tumours that have replaced the entire kidney, radical nephrectomy would be the optimal treatment choice; however, for patients with a small renal mass it is possible to perform nephron-sparing surgery, which can avoid some of the adverse effects associated with radical nephrectomy. Now, the possibility has been raised that a novel imaging biomarker could be used to guide these treatment choices, and thereby optimize therapy and surgical options.

\section{4 ...this information will be tremendously useful in the assessment of renal masses... 77}

Because renal biopsy is only performed in $10-20 \%$ of cases, a noninvasive procedure that could determine the level of treatment needed would be a big step forward. An obvious choice for a technique to achieve this would be PET-CT, and that is the method that was assessed by Chaitanya Divgi and his coauthors in the REDECT trial.

This trial was a phase III, multicentre, open-label assessment of PET-CT imaging using a novel imaging marker. The marker consists of ${ }^{124}$ I-labelled girentuximab, an antibody that binds to carbonic anhydrase IX, which is a cell-surface antigen that is expressed in $>95 \%$ of ccRCC. The study recruited 226 patients with renal cortical tumours to be imaged with both ${ }^{124}$ I-labelled girentuximab PET-CT and contrast-enhanced CT (CECT). Of the patients who received the study drug and the subsequent imaging, 202 went on to have surgery, and 195 of these had all image sets and the relevant histopathology samples available for assessment.

${ }^{124}$ I-labelled girentuximab was well tolerated, with no reports of allergic reactions or of drug intolerance. The most common treatment-related adverse events were headache (4.4\% of patients), nausea ( $1.3 \%$ of patients), diarrhoea, dizziness and hot flashes $(0.9 \%$ of patients each). One grade 3 adverse event was reported to be associated with the study drug (transient liver enzyme increase), although this patient had also received the antibiotic ciprofloxacin.

In terms of detection of ccRCC, the ${ }^{124}$ I-labelled girentuximab PET-CT achieved a sensitivity of $86.2 \%$, and a specificity of $85.9 \%$. In both cases, this rate was significantly better than the rate achieved using CECT (75.5\% and $46.8 \%$ for sensitivity and specificity, respectively). As Divgi points out, "this is the first example of a prospective multicentre study to demonstrate the ability of PET-CT to identify an aggressive cancer phenotype (ccRCC)." He continues: "this information will be tremendously useful in the assessment of renal masses, potentially avoiding unnecessary surgery as well as informing the nature of any surgical procedure."

In addition, for the secondary end points of the study-overall accuracy, positive predictive value and negative predictive value-the estimates for ${ }^{124}$ I-labelled girentuximab PET-CT were consistently higher than achieved with CECT.

The authors point out that a possible limitation of their study was that it required them to recruit presurgical patients, that is patients who were eligible for subsequent surgery. This requirement enabled the comparison of the imaging modalities with each other and with the surgical biopsy data.

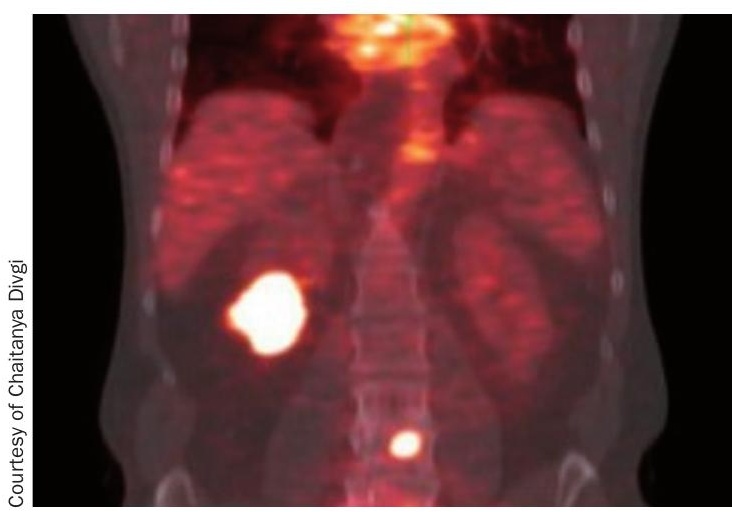

However, ${ }^{124}$ I-labelled girentuximab PET-CT will likely be most useful in determining which patients can be spared from surgery, and those patients in whom surgical biopsy is not possible.

Going forward, Divgi proposes that ${ }^{124}$ I-labelled girentuximab PET-CT "might be used to replace or guide biopsy. Moreover, the extent of disease evaluation would be very accurate in patients with ccRCC, since the imaging identifies an antigen associated with a malignant phenotype, with consequent high sensitivity and specificity." However, for this advance to occur, the imaging biomarker would need to be approved for use by the FDA and other agencies. Divgi says that "although the FDA granted this study a Special Protocol Assessment designation, another phase III might be required for approval."

It is to be hoped that this additional trial can be conducted with high priority and that this advance will be in the clinic soon.

Rebecca Kirk

Original article Divgi, C. R. et al. Positron emission
tomography/computed tomography identification of clear
cell renal cell carcinoma: results from the REDECT trial.
J. Clin. Oncol. doi:10.1200/JC0.2011.41.2445
Further reading Nature Reviews Clinical Oncology.
Focus on Imaging [online], http://www.nature.com/
nrclinonc/focus/imaging/index.html (2012)

